\title{
ON THE CONTINUOUS LINEAR IMAGE OF A BANACH SPACE
}

\author{
R. W. CROSS \\ (Received 22 June 1979) \\ Communicated by W. Moran
}

\begin{abstract}
A subspace of a Banach space is called an operator range if it is the continuous linear image of a Banach space. Operator ranges and operator ideals with fixed range space are investigated. Properties of strictly singular, strictly cosingular, weakly sequentially precompact, and other classes of operators are derived. Perturbation theory and closed semi-Fredholm operators are discussed in the final section.
\end{abstract}

1980 Mathematics subject classification (Amer. Math. Soc.) : Primary 47 A 05; secondary 47 A 53, 47 A 55, 47 D 30, 47 D 40, 46 B 25.

\section{Introduction}

We call a subset $R$ of a Banach space $Y$ an operator range if there exists a Banach space $X$ and a bounded linear operator $T$ defined on $X$ whose range is $R$. If there exists $T \in[Y]$ such that $R(T)=R$, we call $R$ an emdomorphism range. Throughout this paper $X, Y$ and $Z$ will denote infinite dimensional Banach spaces.

Which subspaces of a Banach space are operator ranges, and which are endomorphism ranges? We have the following characterization : A subspace $R$ of a Banach space is an operator range if and only if there exists a stronger norm on $R$ under which $R$ is complete (Proposition 2.1). Obviously every closed subspace is an operator range. However, there exist closed subspaces which are not endomorphism ranges (except in certain Banach spaces like $l_{1}(S)$ and Hilbert space). For example, let $E$ be a separable irreflexive closed subspace of $m$. Then $E$ is not an endomorphism range, since every bounded linear operator from $m$ into a separable space is weakly compact (Grothendieck (1953)). There exist in every infinite dimensional Banach space, subspaces which are not operator ranges; for example, the null space of a 
discontinuous linear functional (for further examples see the remark after Corollary 2.5). We do not know of a characterization of endomorphism ranges.

Every operator range is the image of an $l_{1}(S)$ space; this is because every Banach space is a quotient of such a space. It is moreover clear that there is an $l_{1}(S)$ space which simultaneously maps onto each member of any given finite collection of operator ranges.

If $R$ is an operator range in $Y$, then $Y$ can be embedded in another Banach space in which $R$ becomes an endomorphism range : Let $T \in[X, Y]$ satisfy $R(T)=R$ and define $U(x, y)=(0, T x)$; then $0 \times R$ is an endomorphism range in $X \times Y$.

What happens if the topology of $R$ varies? It can be shown, using the closed graph theorem, that if $T \in[X, Y]$ and $R(T)=R$, then $R$ is an operator range in any Banach space $Z$ containing $R$ whenever $Y$ and $Z$ induce comparable topologies on $R$.

Many of the results of this paper contain as special cases known properties of endomorphism ranges in Hilbert space; these latter are called 'operator ranges' by Fillmore and Williams (1971), 'Julia varieties' by Dixmier (1949) and 'semi-closed subspace' by Foias (cf. Fillmore and Williams (1971)). The results of the first three sections of this paper were previously announced without proof in Cross (1977).

In Section 2 below we develop some of the basic properties of operator ranges; these include properties of sums and intersections, complementation and isomorphism. Section 3 studies operator ideals with fixed range space and variable domain space and includes a result on strictly cosingular operators (Theorem 3.5the proof depends on a result of Vladimirskii (1967)). Consideration of the ideal of weakly sequentially precompact operators shows that if an operator $T$ factors through an almost reflexive space then $R(T)$ is the range of a strictly singular operator. Section 4 discusses perturbation theory and includes applications of the preceding theory.

\section{Operator ranges}

2.1. Proposition. Let $R$ be a linear subspace of the Banach space $Y$. The following properties are equivalent:

(i) $R$ is an operator range;

(ii) $R$ is the domain of a closed operator defined in $Y$;

(iii) there is a norm $\|\cdot\|_{1}$ on $R$ such that $\left(R,\|\cdot\|_{1}\right)$ is a Banach space and $\|y\|_{1} \geqslant\|y\|$ for $y \in R$.

Proof. Assume (i). There exists a Banach space $X$ and $T \in[X, Y]$ such that $R(T)=R$. Then $\hat{T}^{-1}$ satisfies (ii). Next, assume (ii) and let $T \in C(Y, X)$ where $D(T)=R$. Define $\|y\|_{1}=\|y\|+\|T y\|(y \in R)$. Then $\left(R,\|\cdot\|_{1}\right)$ is clearly a Banach space and (iii) follows. Finally, assume (iii). Put $X=\left(R,\|\cdot\|_{1}\right)$ and let $T$ be the 
injection map of $R$ into $Y$ defined by $T y=y$. Then $T \in[X, Y]$ and $R(T)=R$. Since $X$ is complete, (i) follows.

2.2 Proposition. Let $R_{1}$ and $R_{2}$ be operator ranges in $Y$. Then $R_{1}+R_{2}$ and $R_{1} \cap R_{2}$ are operator ranges.

Proof. Let $T_{1} \in\left[X_{1}, Y_{1}\right]$ and $T_{2} \in\left[X_{2}, Y_{2}\right]$ satisfy $R\left(T_{1}\right)=R_{1}, R\left(T_{2}\right)=R_{2}$. Define $T \in\left[X_{1} \times X_{2}, Y\right]$ by $T\left(x_{1}, x_{2}\right)=T_{1} x_{1}+T_{2} x_{2}$. Then $R(T)=R_{1}+R_{2}$.

By proposition 2.1 there exist stronger norms $\|\cdot\|_{1}$ and $\|\cdot\|_{2}$ defined on $R_{1}$ and $R_{2}$ respectively, making $\left(R_{1},\|\cdot\|_{1}\right)$ and $\left(R_{2},\|\cdot\|_{2}\right)$ complete. On $R_{1} \cap R_{2}$ define the norm $\|y\|_{3}=\|y\|_{1}+\|y\|_{2}$. Then $\|y\|_{3} \geqslant\|y\|\left(y \in R_{1} \cap R_{2}\right)$, and it is easily checked that $\left(R_{1} \cap R_{2},\|\cdot\|_{3}\right)$ is complete. Hence by Proposition $2.1, R_{1} \cap R_{2}$ is an operator range.

2.3 Corollary. If $X \times X$ is isomorphic to $X$, then the endomorphism ranges in $X$ have the lattice property of Proposition 2.2.

Part (b) of the following theorem was established for endomorphisms in Hilbert space by Dixmier (1949); see Fillmore and Williams (1971), p. 263.

2.4 TheOREM. Let $R_{1}$ and $R_{2}$ be operator ranges in $Y$ such that $R_{1}+R_{2}$ is closed. Then

(a) if $R_{1} \cap R_{2}$ is closed then $R_{1}$ and $R_{2}$ are closed;

(b) if $R_{1}$ and $R_{2}$ are dense then $R_{1} \cap R_{2}$ is dense.

Proof. (a) Let $T_{1} \in\left[X_{1}, Y\right], T_{2} \in\left(X_{2}, Y\right]$ and $T \in\left[X_{1} \times X_{2}, Y\right]$ be defined as in the proof of Proposition 2.2. Let $P$ be the projection of $X_{1} \times X_{2}$ onto $X_{1} \times 0$ with null space $0 \times X_{2}$. Then $T P=\left(x_{1}, x_{2}\right)=T_{1} x_{1}$ and $T(I-P)\left(x_{1}, x_{2}\right)=T_{2} x_{2}$. Now $T^{-1}\left(R_{1}\right)$ is closed, since $T$ is continuous, $R_{1} \cap R_{2}$ is closed, and $T^{-1}\left(R_{1}\right)=(T(I-P))^{-1}\left(R_{1} \cap R_{2}\right)$. Observing that $T$ has closed range and that $T^{-1}\left(R_{1}\right)$ contains $N(T)$, it follows that $R_{1}$ is closed. Similarly $R_{2}$ is closed.

(b) With $T_{1}, T_{2}$ as above, let $T_{11} \in\left[X_{1} \times 0, Y\right]$ and $T_{21} \in\left[0 \times X_{2}, Y\right]$ be defined by $T_{11}\left(x_{1}, 0\right)=T_{1} x_{1}$ and $T_{21}\left(0, x_{2}\right)=T_{2} x_{2}$. Then $T=T_{11} P+T_{21}(I-P)$ is bounded linear map of $X_{1} \times X_{2}$ onto $R_{1}+R_{2}$. Since $R_{1}+R_{2}$ is closed, $T$ is an open map, and since $R_{1}$ is assumed dense in $Y$, it follows by Jameson (1974), p. 38, that $T^{-1}\left(R_{1}\right)$ is dense in $X_{1} \times X_{2}$. We have $T^{-1}\left(R_{1}\right)=\left\{\left(x_{1}, x_{2}\right): T_{2} x_{2} \in R_{1} \cap R_{2}\right\}$. Also $T_{21}(I-P)\left(T^{-1}\left(R_{1}\right)\right.$ is dense in $R_{2}$ by the continuity of $T_{21}(I-P)$. But

$$
\begin{aligned}
T_{21}(I-P)\left\{\left(x_{1}, x_{2}\right): T_{2} x_{2} \in R_{1} \cap R_{2}\right\} & =T_{21}\left\{\left(0, x_{2}\right): T_{2} x_{2} \in R_{1} \cap R_{2}\right\} \\
& =T_{2}\left\{x_{2} \in X_{2}: T_{2} x_{2} \in R_{1} \cap R_{2}\right\} \subset R_{1} \cap R_{2} .
\end{aligned}
$$

Therefore $R_{1} \cap R_{2}$ is dense in $R_{2}$ and hence in $Y$. 
2.5 COROLLARY. If $R_{1}$ and $R_{2}$ are disjoint complementary operator ranges in $Y$, then $R_{1}$ and $R_{2}$ are closed.

This result yields a construction of subspaces which are not operator ranges : Let $E$ be closed non-complemented subspace of $Y$, and let $P$ be a linear projection of $Y$ onto $E$. Then $N(P)$ is not an operator range (otherwise by Corollary 2.5, $P$ would be bounded).

A special case of Corollary 2.5 is the following well-known property : If the range of a closed operator is complemented, then it is closed (since there is a bounded operator with the same range) (compare Goldberg (1966), Theorem IV.1.12).

Observe that the condition that $R_{1}+R_{2}$ be closed is essential in Theorem 2.4. This is obvious in the case of (a), and in the case of (b) follows from the next proposition.

2.6 Proposition. Let $Y$ be separable. There exists a pair of dense operator ranges $R_{1}$ and $R_{2}$ such that $R_{1} \cap R_{2}=0$.

Proof. There exist endomorphisms $S_{1}, S_{2}$ of $l_{2}$ such that $R\left(S_{1}\right) \cap R\left(S_{2}\right)=0$ with $R\left(S_{1}\right)$ and $R\left(S_{2}\right)$ dense in $l_{2}$ (Fillmore and Williams (1971), p. 273). Let $J$ be a (compact) one-to-one map of $l_{2}$ into $Y$ with dense range (cf. Goldberg and Kruse (1962)), and consider $T_{1}=J S_{1}, T_{2}=J S_{2}$.

A closed operator $T$ is called semi-Fredholm if it has closed range and if either the dimension of $N(T)$ or the codimension of $R(T)$ is finite.

2.7 Proposition. Let $T \in[X, Y]$. If there exists a bounded semi-Fredholm operator $U$ defined either on $X$ or on $X / N(T)$ with range contained in $R(T)$, then $R(T)$ is the sum of $R(U)$ and another closed subspace.

Proof. Let $U \in[X, Y]$ be semi-Fredholm and let $R(T) \supset R(U)$. If codim $R(U)<\infty$ then codim $R(T)<\infty$, and the assertion follows. Suppose, on the other hand, that $\operatorname{dim} N(U)<\infty$, i.e. that $U$ is a $\phi_{+}$-operator. The set of $\phi_{+}$-operators is an open subset of $[X, Y]$ (Brown and Page (1970), p. 328), and therefore $T+\lambda U \in \phi_{+}$for sufficiently large $\lambda$. Then

$$
R(T)=R(T+\lambda U) \subset R(T+\lambda U)+R(U) \subset R(T)
$$

whence $R(T)=R(T+\lambda U)+R(U)$, as required. For the second case consider the operator $\hat{T}: X / N(T) \rightarrow Y$ induced by $T$.

2.8 COROLlaRY. Let $R$ be an endomorphism range in $X$. If $R$ contains a subspace isomorphic to $X$ then $R$ is the sum of two closed subspaces. 
Of particular interest is the case $X=l_{2}$. In this case it follows immediately that $R$ is the orthogonal sum of two subspaces (compare with Fillmore and Williams (1971), Theorem 2.6).

A Banach space $X$ which maps onto $R \subset Y$ under a continuous one-to-one linear map is called the pre-image space of $R$; it is isomorphic to space $\left(R,\|\cdot\|_{1}\right)$ of Proposition 2.1.

2.9 COROLlaRY. Let $R$ be an operator range. If $R$ contains a copy of its pre-image space then $R$ is the sum of two closed subspaces.

2.10 COROLLARY. Let $R$ be an operator range in $l_{1}$ with separable pre-image space. If $R$ contains an infinite dimensional closed subspace then $R$ is the sum of two closed subspaces.

Proof. If $X$ is the pre-image space, there exists a bounded linear map of $l_{1}$ onto $X$ (Jameson (1974), p. 215). Now apply Corollary 2.8, using the fact the $R$ contains a copy of $l_{2}$ (loc. cit., p. 336).

2.11 THEOREM. Let $R_{1}$ and $R_{2}$ be operator ranges in $Y$ such that $R_{1} \cap R_{2}$ has a closed complementary subspace. Then $R_{1}+R_{2}=Y$ if an only if there exist disjoint closed subspaces $M_{1}$ and $M_{2}$ with $M_{1} \subset R_{1}$ and $M_{2} \subset R_{2}$ such that $M_{1} \oplus M_{2}=Y$.

Proof. Suppose that $R_{1}+R_{2}=Y$ and let $E$ be a closed subspace such that $R_{1} \cap R_{2} \oplus E=Y$. Since $R_{1} \cap R_{2}$ is an operator range (Proposition 2.2), it is a closed subspace, by Corollary 2.5. Hence there exists a bounded projection $P$ of $Y$ onto $E$ along $R_{1} \cap R_{2}$. Let $T \in\left[X_{1}, Y\right], T_{2} \in\left[X_{2}, Y\right]$ satisfy $R_{1}=R\left(T_{1}\right), R_{2}=R\left(T_{2}\right)$. Define $A=T_{1} / T_{1}^{-1}(E)$ and $B=T_{2} / T_{1}^{-1}(E)$. We clearly have $R(A) \cap R(B)=0$. If $P y \in P\left(R\left(T_{1}\right)\right)\left(y \in R\left(T_{1}\right)\right)$ then

$$
P y=y-(I-P) y \in R\left(T_{1}\right)+R\left(T_{1}\right) \cap R\left(T_{2}\right) \subset R\left(T_{1}\right) .
$$

Hence $P\left(R\left(T_{1}\right)\right) \subset R\left(T_{1}\right)$ and $P\left(R\left(T_{2}\right)\right) \subset R\left(T_{2}\right)$. Next we have

$$
R(A)=E \cap R\left(T_{1}\right)=P\left(E \cap R\left(T_{1}\right)\right) \subset E \cap P\left(R\left(T_{1}\right)\right)=P\left(R\left(T_{1}\right)\right) \subset E \cap R\left(T_{1}\right) .
$$

Consequently

$$
R(A)=P\left(R\left(T_{1}\right)\right) \quad \text { and } \quad R(B)=P\left(R\left(T_{2}\right)\right)
$$

It follows that

$$
R(A) \oplus R(B)=P\left(R\left(T_{1}\right)+R\left(T_{2}\right)\right)=P(Y)=E .
$$

Let $M_{1}=R(A), M_{2}=R(B)+R_{1} \cap R_{2}$. Clearly $M_{1}+M_{2}=Y$. A straightforward check shows that $M_{1} \cap M_{2}=0$. Hence $Y=M_{1} \oplus M_{2}$. The converse is trivial. 
The above theorem fails unless $R_{1} \cap R_{2}$ is at least closed; as a counterexample, take $R_{1}$ as the range of an infinite dimensional compact operator and $R_{2}=Y$.

2.12 THEOREM. Let $X=c_{0}$ or $l_{p}(1 \leqslant p<\infty)$ and let $E$ be a linear subspace of $X$. Then $E$ contains no closed infinite dimensional subspace if and only if every endomorphism of $X$ with range in $E$ is compact.

Proof. Let $E$ contain no closed infinite dimensional subspace and let $T$ be an endomorphism of $X$ satisfying $R(T) \subset E$. Then $T$ is clearly strictly singular. Since the only two-sided closed ideal in $[X]$ consists of the compact operators by Goldberg, Marcus and Feldman (1967), $T$ is compact. Conversely, suppose $E$ contains an infinite dimensional closed subspace. Then $E$ contains a subspace $G$ isomorphic to $X$ by Whitley (1964), and any isomorphism $T$ of $X$ into $G$ satisfies $R(T) \subset E$ and is not compact.

Theorem 2.12 is a generalization of Theorem 2.5 of Fillmore and Williams (1971).

Two operators $U, T$ are called equivalent if there exist invertible operators $A$ and $B$ such that $T=A U B$.

2.13 Proposition. The operator ranges $R_{1}$ and $R_{2}$ in the Banach space $Y$ are isomorphic if and only if there exist equivalent operators $T_{1}$ and $T_{2}$ such that $R\left(T_{1}\right)=R_{1}$ and $R\left(T_{2}\right)=R_{2}$.

Proof. Let $R_{1}$ and $R_{2}$ be isomorphic. Choose injective bounded operators $T_{1}$ and $T_{2}$ such that $R\left(T_{1}\right)=R_{1}$ and $R\left(T_{2}\right)=R_{2}$. Let $J \in[\bar{R}, Y]$ be an isomorphism such that $J\left(R_{1}\right)=R_{2}$. Then $R\left(J T_{1}\right)=R\left(T_{2}\right)$. The linear map $\left(J T_{1}\right)^{-1} T_{2}$ is a closed injective operator mapping $D\left(T_{2}\right)$ onto $D\left(T_{1}\right)$, and is therefore an invertible isomorphism. Viewing $J$ as an invertible isomorphism of $\bar{R}_{1}$ onto $\bar{R}_{2}$, the factorization $T_{2}=J T_{1}\left(J T_{1}\right)^{-1} T_{2}$ shows that $T_{1}$ and $T_{2}$ are equivalent. The converse is trivial.

Two operator ranges $R_{1}$ and $R_{2}$ (not necessarily situated in the same Banach space) are called similar if they are continuous linear images of each other. Similarity is an equivalence relation on the class of operator ranges. The following result is a consequence of the closed graph theorem.

2.14 Proposition. Similar operator ranges have similar pre-image spaces.

2.15 PROPOSITION. A Banach space Yis the range of a strictly singular operator if and only if $Y$ contains no subspace isomorphic to $l_{1}$.

Proof. Suppose $T \in[X, Y]$ is a strictly singular operator with $R(T)=Y$. If there exists an injective isomorphism $U: l_{1} \rightarrow Y$, then by the lifting property of $l_{1}$ there 
exists $\tilde{U}: l_{1} \rightarrow X$ such that $T \tilde{U}=U$. But then $U$ would be strictly singular which is impossible.

Conversely, suppose that $Y$ contains no subspace isomorphic to $l_{1}$. There exists a set $S$ of suitable cardinality and a quotient map $h$ of $l_{1}(S)$ onto $Y$. Let $M$ be a closed subspace of $l_{1}(S)$ such that $h / M$ is an isomorphism. Since every infinite dimensional closed subspace of $l_{1}(S)$ contains a subspace isomorphic to $l_{1}$ (cf. Lacey and Whitley (1965), p. 2), $M$ must be finite dimensional. Hence $h$ is strictly singular as required.

2.16 COROLLARY. There is no strictly singular operator whose range is an $L_{1}(\mu)$ space.

For a given operator range $R$ let $\|\cdot\|_{1}$ be any norm making $\left(R,\|\cdot\|_{1}\right)$ complete (compare with Proposition 2.1).

2.17 COROLlaRY. Let $R$ be an operator range in $Y$ which is not the range of any strictly singular operator. Then $\left(R,\|\cdot\|_{1}\right)$ contains a subspace isomorphic to $l_{1}$.

PROOF. If $\left(R,\|\cdot\|_{1}\right)$ contains no copy of $l_{1}$ then there exists a bounded strictly singular map $T$ of $X$ onto $\left(R,\|\cdot\|_{1}\right)$, by the theorem. But then $T$ is clearly a bounded strictly singular map of $X$ into $Y$ with range $R$.

The converse of Corollary 3.7 is false; consider the natural injection of $l_{1}$ into $c_{0}$.

\section{Ideals of operators}

In this section we introduce the concept of a $B R$-ideal. It differs from the more usual definitions of ideal in that the range space is kept fixed.

Let $B R(Y)$ denote the class of bounded linear maps with range contained in $Y$. A subclass $J$ of $B R(Y)$ is called a left $B R$-ideal if it satisfies the following two conditions

(i) for each Banach space $X$, the set $J \cap[X, Y]$ is either empty, or is a linear subspace of $[X, Y]$;

(ii) for each $T \in J$ and each operator $V$ satisfying $R(V) \subset D(T)$ we have $T V \in J$.

The subclass $J$ of $B R(Y)$ is called a $B R$-ideal if it satisfies (i) and (ii) and the following :

(iii) for each $T \in J$, if $U \in[Y]$ then $U T \in J$.

A left semi-ideal in $B R(Y)$ is any subclass $J$ satisfying (ii) above.

Let $S$ be a subclass of $B R(Y)$. We call a subspace $R$ of $Y$ an operator range of type $S$ (respectively, of type $\hat{S}$ ) if it is the range of an operator (respectively, an injective operator) belonging to $S$. Clearly, if $S$ is a class of operators satisfying (iii) and $R_{1}$ and $R_{2}$ are similar operator ranges, then $R_{1}$ is of type $S$ if and only if $R_{2}$ is of type $S$. 
Examples of $B R$-ideals are: compact, strictly singular, strictly cosingular, completely continuous and weakly compact operators. A further example is given below.

If $J$ is a $B R$-ideal in $B R(Y)$ then $J \cap[Y]$ is a two-sided ideal in $[Y]$ in the usual Banach-algebra sense (unless empty). Conversely, every ideal in $[Y]$ is a $B R$-ideal.

3.1 THEOREM. Let $J$ be a BR-ideal and let $R_{1}$ and $R_{2}$ be operator ranges of type $J$. Then $R_{1}+\mathbf{R}_{2}$ and $R_{1} \cap \mathbf{R}_{2}$ are operator ranges of type $J$. A similar result holds for left BR-ideals.

Proof. Let $T_{1} \in\left[X_{1}, Y\right] ; T_{2} \in\left[X_{2}, Y\right], T$ and $P$ be defined as in the proof of Theorem 2.4, and suppose that $T_{1}$ and $T_{2}$ are members of $J$. Define $i \in\left[X_{1}, X_{1} \times X_{2}\right]$ by $i\left(x_{1}\right)=\left(x_{1}, 0\right)$. Then $T_{1}=T P i$ and $T P=(T P i)\left(i^{-1} P\right) \in J$. Similarly, $T(I-P) \in J$, and therefore $T=T P+T(I-P)$ is in $J$. Since $R_{1}+R_{2}=R(T)$, it is an operator range of type $J$.

Let $T_{0}=T / T^{-1}\left(R_{1} \cap R_{2}\right)$ and let $X$ be the linear space $T^{-1}\left(R_{1} \cap R_{2}\right)$ normed by $\left\|\left(x_{1}, x_{2}\right)\right\|_{X}=\left\|x_{1}\right\|+\left\|x_{2}\right\|+\left\|T_{0}\left(x_{1}, x_{2}\right)\right\|_{1}$ where the norm \|\|$_{1}$ is any norm making $R_{1} \cap R_{2}$ complete (compare with Proposition 2.1).

To show that $X$ is complete, let $\left(x_{1}^{n}, x_{2}^{n}\right)$ be a cauchy sequence in $X$. Then $x_{1}^{n} \rightarrow x_{1}$ and $x_{2}^{n} \rightarrow x_{2}$ for some $x_{1} \in X_{1}, x_{2} \in X_{2}$. Hence

$$
T\left(x_{1}^{n}, x_{2}^{n}\right)=T_{1} x_{1}^{n}+T_{2} x_{2}^{n} \rightarrow T_{1} x_{1}+T_{2} x_{2}=T\left(x_{1}, x_{2}\right) .
$$

Since $R_{1} \cap R_{2}$ is complete in the norm $\|\cdot\|_{1}$ which is stronger than the $Y$-norm, there exists $y \in R_{1} \cap R_{2} \quad$ such that $\left\|T_{1} x_{1}^{n}+T_{2} x_{2}^{n}-y\right\|_{1} \rightarrow 0$ which implies $y=T_{1} x_{1}+T_{2} x_{2}$. Hence $\left(x_{1}, x_{2}\right) \in X$ as required. Obviously $T_{0} \in[X, Y]$.

It only remains to show that $T_{0} \in J$. Let $j: X \rightarrow X_{1} \times X_{2}$ be the injection map. It is clearly continuous, and since $T_{0}=T j$, it follows that $T_{0} \in J$.

3.2 Proposition. Let $J$ be a left semi-ideal in $B R(Y)$ and let $R$ be an operator range of type $\hat{J}$. If $T \in B R(Y)$ and satisfies $R(T) \subset R$ then $T \in J$.

Proor. Let $A \in[X, Y]$ be injective and such that $A \in J$ and $R(A)=R$. Let $T \in[Z, Y]$ satisfy $R(T) \subset R$. The operator $A^{-1} T$ is an everywhere defined closed operator and hence $A^{-1} T \in[Z, X]$. Therefore $T=A A^{-1} T \in J$.

3.3 Remarks. (a) Let $J$ consist of either the compact operators, the weakly compact operators, or the strictly cosingular operators. It is easily verified that $T \in J$ if and only if $\hat{T} \in J$. Thus an operator range is of type $J$ if and only if it is of type $\hat{J}$. Consequently, in view of Proposition 3.2, if the range of an operator $T$ is contained in the range of an operator belonging to the class $J$, then $T$ is itself in the class $J$. For 
example, if the range of $T$ is contained in the range of a strictly cosingular operator then $T$ is strictly cosingular.

(b) Let $J$ be the $B R$-ideal of completely continous operators in $B R(Y)$ and let $R$ be an operator range in $Y$. Then $R$ is the image of $l_{1}(S)$, since $l_{1}(S)$ maps onto any preimage of $R$. Since every operator defined on $l_{1}(S)$ is completely continuous, it follows that every operator range $R$ is of type $J$ in this case.

A Banach space $Y$ is called an $h$-space (compare Whitley (1964)) if every closed infinite dimensional subspace of $Y$ contains a subspace isomorphic to $Y$ and complemented in $Y$. Examples are $c_{0}$ and $l_{p}(1 \leqslant p<\infty)$. It is shown in Whitley (1964) that if $X$ is an $h$-space then the greatest ideal in $[X]$ is the ideal of strictly singular operators. This is a consequence of the following result :

3.4 Proposition. Let Ybe an $h$-space. Then the largest proper BR-ideal in BR(Y) consists of the strictly singular operators.

Proof. The proof is a modification of that of (Whitley (1964), Theorem 6.2); we omit the details.

3.5 THEOREM. Let $R_{1}$ and $R_{2}$ be operator ranges satisfying $R_{1}+R_{2}=Y$. If $R_{2}$ is the range of a strictly cosingular operator, then $R_{1}$ is of finite codimension in $Y$.

Proof. Let $T_{1} \in\left[X_{1}, Y\right]$ and $T_{2} \in\left[X_{2}, Y\right]$ satisfy $R\left(T_{1}\right)=R_{1}, R\left(T_{2}\right)=R_{2}$, where $T_{2}$ is strictly cosingular. Let $T \in\left[X_{1} \times X_{2}, Y\right]$ be defined by $T\left(x_{1}, x_{2}\right)=T_{1} x_{1}+T_{2} x_{2}$, and let $P$ be the projection defined by $P\left(x_{1}, x_{2}\right)=\left(x_{1}, 0\right)$. Then $T(I-P)$ is strictly cosingular since $R(T(I-P))=R\left(T_{2}\right)$ (Remarks 3.3(a)). Since $T$ is a $\varphi_{-}$-operator, and since $\varphi_{-}$is stable under the addition of a strictly cosingular operator by Vladimirskii (1967), it follows that $T P=T-T(I-P)$ is a $\varphi_{-}$-operator, that is, $R_{1}=R(T P)$ is of finite codimension.

A special case of Theorem 3.5, for compact endomorphisms in Hilbert space, is given in Fillmore and Williams (1971), p. 263.

The property of Theorem 3.5 fails for strictly singular operators-consider any map of $l_{1}$ onto $l_{2}$.

An operator $T \in[X, Y]$ is said to be weakly sequentially precompact (WSP) if whenever $x_{n}$ is a bounded sequence in $X, T x_{n}$ has a weak cauchy subsequence. For example, if either $X$ or $Y$ is almost reflexive (cf. Lacey and Whitley (1965)) then $T$ is $W S P$. The class of WSP operators in $B R(Y)$ is a BR-ideal. It is easily verified that $T$ is $W S P$ if and only if $\hat{T}$ is $W S P$. Hence, by Proposition 3.2, if the range of an operator $T$ is contained in the range of a $W S P$ operator then $T$ is $W S P$. For example, let $A$ be defined on $c_{0}$ and let $R(T) \subset R(A)$. Then $T$ is $W S P$. 
3.6 Proposition. Let $T \in[X, Y]$ be WSP but not strictly singular. Then both $X$ and $Y$ contain almost reflexive infinite dimensional subspaces.

3.7 Proposition (Lacy and Whitley (1965)). If $T \in[X, Y]$ is completely continuous but not strictly singular then both $X$ and $Y$ contain an infinitive dimensional closed subspace in which weak and norm sequential convergence coincide.

Propositions 3.6 and 3.7 together show that if an operator is both completely continuous and WSP then it is strictly singular. Hence if an operator range is of WSP type then it is of strictly singular type (cf. Remarks 3.3(b)). In particular, if an operator $T$ factors through an almost reflexive space then $R(T)$ is of strictly singular type.

\section{Perturbation theory}

We denote by $L X, Y)$ the set of linear transformations $T$ defined on a linear subspace $D(T)$ of $X$ with range $R(T)$ contained in $Y$. The closed operators in $L(X, Y)$ are denoted by $C(X, Y)$ and the bounded (everywhere defined) operators by $[X, Y]$. If $T \in L(X, Y)$, we write ${ }^{*} D(T)$ for the subspace $D(T)$ equipped with the norm $\|x\|+\|T x\|$. The class $B R(Y)$ contains all the closed operators with range in $Y$ in the following sense : if $T \in C(X, Y)$ then ${ }^{\sharp} D(T)$ is a Banach space and $T \in\left[{ }^{\sharp} D(T), Y\right]$.

A subset $E$ of $L(X, Y)$ is called uniformly open if for each $T \in E$ there exists $\delta>0$ such that $T+U \in E$ whenever $U$ is a bounded operator in $[X, Y]$ satisfying $\|U\|<\delta$. $E$ is called uniformly closed if its complement is uniformly open. The uniformly open sets define a topology on $L(X, Y)$ which induces the norm topology on $[X, Y]$.

An operator $T \in L X, Y)$ is called strictly singular if there is no infinite dimensional subspace $M$ of $D(T)$ such that the restriction $T / M$ of $T$ to $M$ is an isomorphism (into). It is called strictly cosingular if there is no infinite dimensional Banach space $Z$ such that $U T$ is surjective for some surjective $U \in[Y, Z]$. (These definitions are generalizations of those of Pelczynski (1965) for bounded operators.) A normally solvable operator is a closed operator whose range is closed.

In $C(X, Y)$ we define the classes $\varphi_{i}^{C}(X, Y)$, abbreviated $\varphi_{i}^{c}, i=1,2,3,4,5$, as follows :

$$
\begin{aligned}
& T \in \varphi_{1}^{C}(X, Y) \Leftrightarrow \alpha(T)<\infty \text { and } \beta(T)<\infty, \\
& T \in \varphi_{2}^{c}(X, Y) \Leftrightarrow T \in \varphi_{+}^{c}(X, Y) \text { and } R(T) \text { is complemented, } \\
& T \in \varphi_{3}^{c}(X, Y) \Leftrightarrow T \in \varphi_{-}^{C}(X, Y) \text { and } N(T) \text { is complemented in } D(T), \\
& T \in \varphi_{4}^{c}(X, Y) \Leftrightarrow T \in \varphi_{+}^{c}(X, Y) \backslash\left\{\varphi_{1}^{C}(X, Y) \cup \varphi_{2}^{C}(X, Y)\right\}, \\
& T \in \varphi_{5}^{c}(X, Y) \Leftrightarrow T \in \varphi_{-}^{c}(X, Y) \backslash\left\{\varphi_{1}^{C}(X, Y) \cup \varphi_{3}^{C}(X, Y)\right\},
\end{aligned}
$$


where $\varphi_{+}^{c}(X, Y)\left(\varphi_{-}^{c}(X, Y)\right)$ consists of normally solvable operators with $\alpha(T)<\infty$ (respectively, $\beta(T)<\infty$ ). Observe that if $R(T)$ is complemented, in particular if $\beta(T)<\infty$, then $R(T)$ is closed (Corollary 2.5).

In $[X, Y]$ we define the corresponding classes $\varphi_{i}(X, Y)$ (abbreviated $\left.\varphi_{i}\right)$ by setting $\varphi_{i}=\varphi_{i}^{c} \cap[X, Y]$. For notational convenience we write

$$
\left(\varphi_{1}^{\mathrm{C}} \cup \varphi_{2}^{\mathrm{C}}\right)(X, Y),\left(\varphi_{1} \cup \varphi_{2} \cup \varphi_{3}\right)(X, Y), \ldots
$$

for the unions

$$
\varphi_{1}^{C}(X, Y) \cup \varphi_{2}^{C}(X, Y), \varphi_{1}(X, Y) \cup \varphi_{2}(X, Y) \cup \varphi_{3}(X, Y), \ldots
$$

and these will be abbreviated $\varphi_{1}^{C} \cup \varphi_{2}^{C}, \varphi_{1} \cup \varphi_{2} \cup \varphi_{3}, \ldots$. We also write $\varphi_{i}(X)$ and $\varphi_{i}^{C}(X)$ for $\varphi_{i}(X, X)$ and $\varphi_{i}^{C}(X, X)$ respectively. The sets $\varphi_{i}\left(\varphi_{i}^{C}\right)$ form a disjoint partition of the bounded (respectively, closed) semi-Fredholm operators. Observe that if $U, T \in \varphi_{i}$ then $U T \in \varphi_{i}$ whenever $U T$ is defined $(1 \leqslant i \leqslant 5)$. We also have for each $i$

$$
T \in \varphi_{i}(X, Y) \Leftrightarrow T \in \varphi_{i}^{C}\left({ }^{*} D(T), Y\right) .
$$

Hence using the results of Nieto (1968) for bounded operators we obtain :

4.1 Proposition. Let $T \in C(X, Y)$. Then $T \in \varphi_{1}^{C} \cup \varphi_{3}^{c}\left(T \in \varphi_{1}^{C} \cup \varphi_{2}^{C}\right)$ if and only if there exists $V \in[Y, X]$ such that $R(V) \subset D(T)$, and $T V=I+F$ (respectively, $V T=I+F$ on $D(T)$ ), where $F$ is an everywhere defined bounded operator of finite dimensional range.

4.2 Proposition. The classes $\varphi_{1}^{c}, \varphi_{2}^{c}, \varphi_{3}^{c}, \varphi_{2}^{c} \cup \varphi_{4}^{c}$ and $\varphi_{3}^{c} \cup \varphi_{5}^{c}$ are uniformly open sets.

Proof. First we show that $\varphi_{1} \cup \varphi_{2}$ is open. Let $T \in \varphi_{1} \cup \varphi_{2}$. By Proposition 4.1 there exists $V \in[Y, X]$ such that $V T=I+F$ where $F$ has finite dimensional range. But $\varphi_{1}$ is open by Brown and Page (1970), p. 331. Hence for $U \in[X, Y]$ with $\|U\|$ sufficiently small, $V(T+U)=I+F+V U \in \varphi_{1}(X)$. But then $V_{1} \in[X]$ exists with $V_{1} V(T+U)=I+F_{1}$ where $F_{1}$ has finite dimensional range, and this implies (cf. 4.1) that $T+U \in \varphi_{1} \cup \varphi_{2}$ as required. Since $\varphi_{2} \cup \varphi_{4}$ is open (loc. cit., p. 328), it follows that $\varphi_{2}$ is open. A similar argument shows that $\varphi_{3}$ and $\varphi_{3} \cup \varphi_{5}$ are open.

To show that $\varphi_{1}^{c} \cup \varphi_{2}^{C}$ is uniformly open, let $T \in \varphi_{1}^{c} \cup \varphi_{2}^{C}$. Then

$$
T \in\left(\varphi_{1} \cup \varphi_{2}\right)\left({ }^{*} D(\bar{T}), Y\right) .
$$

Consequently, by the first part, there exists a $\delta$-neighbourhood of $T$ contained in $\left(\varphi_{1} \cup \varphi_{2}\right)\left({ }^{*} D(T), Y\right)$. Let $U \in[X, Y]$ satisfy $\|U\|<\delta$. Since $\|U\| *_{D(n)} \leqslant\|U\|<\delta$, it follows that

$$
T+U \in\left(\varphi_{1} \cup \varphi_{2}\right)\left({ }^{*} D(T), Y\right)
$$

whence $T+U \in \varphi_{1}^{C} \cup \varphi_{2}^{C}$ as required. The remaining verifications are similar.

An interesting (and apparently unsolved) problem is whether or not the sets $\varphi_{4}(X)$ and $\varphi_{5}(X)$ are open. 
Let $S$ be an arbitrary subset of $L(X, Y)$. The set $P(X)$ of admissible perturbations of $S$ is defined as

$$
P(S)=\{A \in L(X, Y): A+T \in S \text { whenever } T \in S \text { and } D(T) \subset D(A)\} .
$$

If $M$ is a subset of $P(S)$ we say that $S$ is stable under perturbation by operators in $M$.

The condition $D(T) \subset D(A)$ cannot be meaningfully relaxed in the definition of $P(S)$. For example suppose $T$ is a semi-Fredholm operator (bounded or unbounded) with $\alpha(T)=\infty$. Let $D(T)=N(T)$ and let $A \in[N(T), Y]$ be compact with non-closed range. Then $T+A$ does not have closed range, and in particular is not semiFredholm.

The following are some easily proved facts concerning $P(S): P(S)$ is closed under finite sums and is topologically closed if $S$ is uniformly open. If $S$ is stable under perturbation of given type (for example, compact perturbation) then so is the uniform closure $\bar{S}$; in particular $P(S) \subset P(\bar{S})$. If $S^{\prime}$ is complement of $S$, then $P(S)=P\left(S^{\prime}\right)$. If $S$ is non-empty and closed under scalar multiplication, then $\bar{S} \supset P(\bar{S})$, making $P(S)$ a subset of the boundary of $S$, and $S \cap[X, Y]$ is a subspace of $[X, Y]$ if and only if $S \cap[X, Y]=P(S) \cap[X, Y]$.

A subset $S$ of $L(X, Y)$ is called automorphism invariant if for each $T \in S$, we have $T U \in S$ and $V T \in S$ whenever $U$ and $V$ are automorphisms of $X$ and $Y$ respectively.

4.3 Proposition. Let $S$ be an automorphism invariant subset of $L(X, Y)$. Then $[Y]_{0} P(S) \subset P(S)$ and $P(S)_{0}[X] \subset P(S)$.

Proof. We show first that $P(S)$ is automorphism invariant. Let $A \in P(S)$ and let $U$ be an automorphism of $X$. If $T \in S$ and $D(T) \subset D(A U)$, we have $A+T U^{-1} \in S$ since $D\left(T U^{-1}\right)=U D(T) \subset D(A)$, and therefore $A U+T \in S$ since $S$ is automorphism invariant. A similar argument shows that if $V$ is an automorphism of $Y$ then $V A \in P(S)$. Thus $P(S)$ is automorphism invariant. In particular, if $c$ is a non-zero scalar then $c A \in P(S)$, while if $c=0, c A \in P(S)$ trivially.

Now let $A \in P(S)$ and $B \in[Y]$. Since the set of automorphisms of a Banach space is open (cf. Jameson (1974), p. 175) there exists $\lambda \neq 0$ such that $I+\lambda B$ is an automorphism. Then by the properties established above, $(I+\lambda B) A \in P(S)$ and therefore $B A \in P(S)$. Thus $[Y]_{0} P(S) \subset P(S)$. Similarly $P(S)_{0}[X] \subset P(S)$.

4.4 COROLLARY. A subset of $[X]$ is an automorphism invariant subspace if and only if it is an ideal.

4.5 Corollary. The classes $P\left(\varphi_{i}^{\mathrm{C}}(X)\right) \cap[X]$ and $P\left(\varphi_{i}(X)\right)$ are ideals.

4.6 Proposition. Let $S \subset L(X, Y)$ be either uniformly open or uniformly closed and consist of normally solvable operators. If $S$ is stable under perturbation by bounded 
operators of finite dimensional range then $S$ consists entirely of semi-Fredholm operators.

Proof. The result can be deduced from the proof of Goldberg (1966), V. 2.6.

4.7 Proposition. Let $A$ be a closed operator. Then $A \in P\left(\varphi_{-}^{c}\right) \Rightarrow \hat{A} \in P\left(\varphi_{-}^{c}\right)$.

Proof. Let $A \in P\left(\varphi_{-}^{c}(X, Y)\right)$. Then $A \in\left[{ }^{\sharp} D(A), Y\right]$ and $A=\hat{A} h$ where $h$ is surjective. Then $h$ is in $\varphi_{-}^{c}$, since $A$ is closed. Suppose $\hat{A} \notin P\left(\varphi_{-}^{c}\right)$. Then there exists $T \in \varphi_{-}^{c}(X / N(A), Y)$ such that $D(T) \subset D(\hat{A})$ and $T+\hat{A} \notin \varphi_{-}^{c}$. However $T h \in \varphi_{-}^{c}$ and $D(T h)=h^{-1}(D(T)) \subset D(A), \quad$ and therefore $\quad(T+\hat{A}) h=T h+A \in \varphi_{-} \quad$ But $R((T+\hat{A}) h) \subset R(T+\hat{A})$, which implies $T+\hat{A} \in \varphi_{-}^{c}$. This contradiction shows that $\hat{A} \in P\left(\varphi_{-}^{\mathrm{C}}\right)$.

4.8 THEOREM. (a) The classes $\varphi_{1}^{c}, \varphi_{2}^{c}, \varphi_{3}^{c}$ and $\varphi_{4}^{c}$ are stable under perturbation by closed strictly singular operators.

(b) The classes $\varphi_{1}^{\mathrm{C}}, \varphi_{2}^{\mathrm{C}}, \varphi_{3}^{\mathrm{C}}$ and $\varphi_{5}^{\mathrm{C}}$ are stable under perturbation by closed strictly cosingular operators.

Proor. (a) The stability of the classes $\varphi_{i}$ under bounded strictly singular perturbation for $i=1,2,3,4$ follows from known results. Indeed, $\varphi_{1}$ and $\varphi_{+}$are stable (Goldberg (1966), V.2.1), the stability of $\varphi_{1} \cup \varphi_{2}$ follows from Lin (1974), Theorem 1, and taking the complement of $\varphi_{1} \cup \varphi_{2}$ in $\varphi_{+}$shows that $\varphi_{4}$ is stable. The stability of $\varphi_{1} \cup \varphi_{3}$ (and hence of $\varphi_{3}$ ) follows again from Lin (1974), Theorem 2.

We complete the proof of (a) by showing that $\varphi_{i}^{c}$ is stable under closed strictly singular perturbation for those values of $i$ for which $\varphi_{i}$ is stable under bounded strictly singular perturbation.

Let $T \in \varphi_{i}^{C}(i=1,2,3,4)$ and let $A \in C(X, Y)$ be strictly singular with $D(T) \subset D(A)$. Let $E$ be $D(T)$ with the norm $\|x\|_{E}=\|x\|+\|T x\|+\|A x\|$. Then because $T$ and $A$ are closed, $E$ is complete. It follows that $E$ and ${ }^{*} D(T)$ are isomorphic. Accordingly $T \in \varphi_{i}^{C}(D(T), Y)=\varphi_{i}(E, Y)$. But $A \in[E, Y]$ and it is clear that $A$ is strictly singular in $[E, Y]$. Consequently $T+A \in \varphi_{i}(E, Y)$ which implies that $T+A \in \varphi_{i}^{C}$ as required.

(b) The stability of $\varphi_{-}$under bounded strictly cosingular perturbation is proved in Pelczynski (1965). Since $P\left(\varphi_{-}\right) \subset P\left(\varphi_{1}\right)$ (Gohberg, Marcus and Feldman (1967), p. 69), the stability of $\varphi_{1}$ follows.

To show that $\varphi_{5}$ is stable, let $T \in \varphi_{5}$ and let $A$ be a bounded strictly cosingular operator. Suppose $T+A \notin \varphi_{5}$. Then, because $\varphi_{-}$is stable, we have $T+A \in \varphi_{1} \cup \varphi_{3}$, and consequently there exists $U \in[Y, X]$ and a bounded operator $F \in[Y]$ of finite dimensional range such that $(T+A) U=I+F$ (Proposition 4.1). Thus $T U=I+F-A U$. But $I+F \in \varphi_{1}$, since $\varphi_{1}$ is stable under finite dimensional perturbation. Also $A U$ is strictly cosingular by Pelczynski (1965), p. 32. Hence 
$T U=I+F-A U \in \varphi_{1}$, by an earlier remark. But $T U \in \varphi_{1}$ implies $T U V=I+F_{1}$ for some $V \in[Y]$ where $F_{1}$ has finite dimensional range, and hence (Proposition 4.1) $T \in \varphi_{1} \cup \varphi_{3}$, a contradiction. Therefore $\varphi_{5}$ is stable. The stability of $\varphi_{3}$ follows by complementation in $\varphi_{1}$.

To show that $\varphi_{2}$ is stable, let $T \in \varphi_{2}$. Then $S T=I+F$, where $S \in[Y, X]$ and $F$ has finite dimensional range. Let $A \in[X, Y]$ be strictly cosingular. Then by Pelczynski (1965) $S A$ is strictly cosingular, and since $I+F \in \varphi_{1}$, we have $S(T+A)=I+F+S A \in \varphi_{1}$. It now follows by an argument similar to the previous that $T+A \in \varphi_{2}$.

The stability of the classes $\varphi_{i}^{C}(i=1,2,3,5)$ can now be proved by using the same technique as in (a).

4.9 Remarks. (1) The stability of $\varphi_{1}^{c}$ under bounded strictly cosingular perturbation, for the case when $D(T)$ is dense in $X$, has been proved in Israel (1974). Our arguments show that the results therein remain valid without any restriction on $D(T)$.

(2) In general $\varphi_{5}$ is not stable under strictly singular perturbation. Let $T \in\left[l_{1} l_{2}\right]$ be surjective. Then $T$ is strictly singular and is easily seen to have no right inverse. Therefore $T \in \varphi_{5}$. But then $T-T=0$ which is not in $\varphi_{5}$.

(3) The following example shows that in general $\varphi_{4}$ is not stable under strictly cosingular perturbation : With $T$ as in (2) above, $T^{*}$ is an injective isomorphism of $l_{2}$ into $m$. Then $T^{*}$ is weakly compact by Goldberg (1966), p. 88, and since $m$ has the Dunford-Pettis property, $T^{*}$ is strictly cosingular by Pelczynski (1965), p. 244. Then $T^{*} \in \varphi_{4}$, while $T^{*}-T^{*}=0$.

Let $T \in C(X, Y)$. If there exists $U \in[Y, X]$ such that $T U=I+F$, where $F$ is a bounded operator of finite dimensional range, then $U$ is called a bounded rightregularizer of $T$ (cf. Lin (1974)).

4.10 THEOREM. The set of closed operators in $L(X, Y)$ having a bounded rightregularizer with closed range is stable under bounded strictly singular and bounded strictly cosingular perturbation.

Proof. The strictly singular case is treated in Lin (1974). Here we deal with the strictly cosingular case. Let $T \in C(X, Y)$, let $U$ be a bounded right-regularizer of $T$ with closed range, let $T U=I+F$, and let $A \in[X, Y]$ be strictly cosingular. Then $(T+A) U=I+F+A U \in \varphi_{1}^{c}$ by Theorem 4.8. Therefore by Proposition 4.1 there exists $U_{1} \in \varphi_{1}(Y)$ such that $(T+A) U U_{1}=I+F_{1}$ where $F_{1}$ is bounded and of finite dimensional range. Obviously $U U_{1}$ has closed range. Therefore $U U_{1}$ is the required right-regularizer of $T+A$. 
Theorems 4.8 and 4.10 together show that the results of Lin (1974) are also valid for bounded strictly cosingular operators in place of (bounded) strictly singular operators.

4.11 Proposition. Let $J$ be a left ideal in $[X]$ and let $R$ be the range of an injective operator in $J$. If the operator $T \in[X]$ satisfies $R(T) \subset R$ then $T \in J$.

Proof. Similar to the proof of Proposition 3.2.

4.12 TheORem. Let $X \times X$ be isomorphic to $X$, and let $S$ be an automorphism invariant subset of $[X]$ containing the surjectice endomorphisms. Suppose that $R_{1}$ and $R_{2}$ are endomorphism ranges satisfying $R_{1}+R_{2}=X$. If $R_{2}$ is contained in the range of an injective operator in the class $P(S)$ then $R_{1}$ is of type $S$.

Proof. By Proposition 4.3, $P(S)$ is an ideal in $[X]$. Let $T_{1}, T_{2} \in[X]$ satisfy $R\left(T_{1}\right)=R_{1}$ and $R\left(T_{2}\right)=R_{2}$. By Proposition 4.11, $T_{2} \in P(S)$. Let $j$ be an isomorphism of $X \times X$ onto $X$ and let $T \in[X]$ be defined by $T j\left(x_{1}, x_{2}\right)=T_{1} x_{1}+T_{2} x_{2}$. Let $P$ be defined by $P\left(x_{1}, x_{2}\right)=\left(x_{1}, 0\right)$. Then $R\left(T_{2}\right)=R\left(T j(I-P) j^{-1}\right)$, and hence by Proposition 4.11, $T j(I-P) j^{-1} \in P(S)$. Since $T$ is surjective, $T \in S$ and therefore $T j P j^{-1}=T-T j(I-P) j^{-1} \in S$. Since $R\left(T j P j^{-1}\right)=R\left(T_{1}\right)$ the result follows.

4.13 Corollary. Let $X \times X$ be isomorphic to $X$ and let $R_{1}$ and $R_{2}$ be endomorphism ranges satsifying $R_{1}+R_{2}=X$. If $R_{2}$ is contained in the range of an injective strictly singular endomorphism then $R_{1}$ has finite codimension in $X$.

Proof. Take $S=\varphi_{1}(X)$ in the theorem.

\section{References}

A. L. Brown and A. Page (1970), Elements of functional analysis (Van Nostrand-Reinhold, London).

R. W. Cross (1977), 'The range of a linear operator', Math. Colloq. Univ. Cape Town 11, 135-143.

J. Dixmier (1949), 'Etude sur les variétés et les opérateurs de Julia', Bull. Soc. Math. France 77, 11-101.

P. A. Fillmore and J. F. Williams (1971), 'On operator ranges', Advances in Math. 7, 254-281.

I. C. Gohberg, A. S. Marcus and I. Feldman (1967), 'Normally solvable operators and ideals associated with them', Amer. Math. Soc. Translations (Ser. 2) 61, 63-84.

S. Goldberg (1966), Unbounded linear operators (McGraw-Hill, New York).

S. Goldberg and A. H. Kruse (1962), 'The existence of compact linear maps between Banach spaces', Proc. Amer. Math. Soc. (5) 13, 808-811.

A. Grothendieck (1953), 'Sur les applications linéaires faiblement compacte d'espaces du type $C(K)$ ', Canad. J. Math. 5, 129-173.

R. B. Israel (1974), 'Perturbations of Fredholm operators', Studia Math. 52, 1-8.

G. J. O. Jameson (1974), Topology and normed spaces (Chapman and Hall, London). 
E. Lacey and R. J. Whitley (1965), 'Conditions under which all the bounded linear maps are compact', Math. Annalen 158, 1-5.

C.-S. Lin (1974), 'Regularizers of closed operators', Canad. Math. Bull. (1) 17, 67-71.

J. I. Nieto (1968), 'On Fredholm operators and the essential spectrum of singular integral operators', Math. Ann. 178, 62-77.

A. Pelczynski (1965), 'Strictly singular and strictly cosingular operators in $L(v)$ spaces', Bull. Acad. Polon. Sci. Sér. Sci. Math. Astronom. Phy. (1) 13, 37-41.

Ju. N. Vladimirskii (1967), 'Strictly cosingular operators', Sov. Math. Doklady 8, 739-740.

R. J. Whitley (1964), 'Strictly singular operators and their conjugates', Trans. Amer. Math. Soc. 113, 252-261.

\section{University of Cape Town}

Rondebosch

South Africa 